\title{
Enhancing cytotoxic chemotherapy response through targeted BET bromodomain inhibition in preclinical pancreatic cancer models
}

\author{
Sandeep Singh, Ross McCauley, Johann R. Schwarz, Roderich Schwarz, Niranjan \\ Awasthi
}

Department of Surgery, Indiana University School of Medicine - South Bend

\section{Background and Hypothesis:}

Pancreatic ductal adenocarcinoma (PDAC) has a poor prognosis and the standard of care regimen, nab-paclitaxel (NPT) plus gemcitabine (Gem), leads to a dismal 8.5 months median survival. Targeted inhibition of Bromodomain and Extra-Terminal (BET) protein is currently under investigation for several cancers. We hypothesize that BET protein pathway inhibition by iBet-762 will enhance cytotoxic chemotherapy response in PDAC.

\section{Experimental Design:}

In vitro cell proliferation assays were performed using WST-1 reagent. Protein expressions were determined by Western Blot analysis. In vivo animal survival and tumor growth experiments were performed in NOD-SCID mice.

\section{Results:}

Inhibition in cell proliferation in human PDAC cells at $1 \mu \mathrm{M}$ concentration in NPT+Gem, iBET-762, and NPT+Gem+iBet762 was $64 \%, 27 \%, 76 \%$ in AsPC-1; $43 \%, 13 \%, 69 \%$ in Panc-1; and $42 \%, 51 \%, 75 \%$ in MIA PaCa cells. iBET-762 decreased oncogenic proteins c-Myc, $\beta$-catenin, Vimentin, and P-AKT while apoptosis related proteins such as cleaved PARP-1 and cleaved caspase- 3 and cell cycle inhibitors proteins P21 \& P27 were increased. In a peritoneal dissemination model, median animal survival compared to control (21 days) was increased after therapy with NPT+Gem (33 days, a $57 \%$ increase), iBet-762 (30 days, a 43\% increase) and NPT+Gem+iBET-762 (44 days, a $110 \%$ increase). Effect of iBET-762 in combination with chemotherapy on local tumor growth is currently underway.

\section{Conclusion and Potential Impact:}

These findings suggest that the effects of standard chemotherapy can be enhanced through specific inhibition of BET proteins activity, and supports the clinical application of iBET-762 in combination with standard chemotherapy in PDAC patients. 